\title{
Peritoneum patch repair in oncologic major resections - An autolog alternative
}

\author{
Catarina Aguiar*, Emanuel Vigia, Ana Marta Nobre, Luis Bicho, Edite Filipe and Jorge Paulino \\ CHBPT, Centro Hepato-Bilio-Pancreatico e Transplantação, Centro Hospitalar Lisboa Central, Lisbon, Portugal
}

\section{Introduction}

At diagnosis only $10-20 \%$ of pancreatic cancer patients present with primarily resectable disease; $20 \%$ have locally advanced unresectable or borderline resectable tumors and $60 \%$ have metastatic disease [1].

Some of resectable are borderline resectable at surgery [2]. Venous resections are frequent during hepatopancreatobiliary surgery and a rapidly available graft may be needed, especially if it had not been planned preoperatively [3].

The evaluation of tumor extension to major vessels involved in the tumor mass, such as the inferior vena cava, superior mesenteric vein or portal vein, may not be a criterion of non resectability and lead to excision of the tumor mass with vascular resection [4]. This reconstruction may imply the need for prosthetic material if the length of autologous vessels is not sufficient, which is not the most desirable option. The surgeon's experience in vascular reconstruction is of great importance for surgical success and postoperative morbidity.

Autologous peritoneal patch or peritoneal tube venous reconstruction is rarely used despite its advantages compared to synthetic implants. Prosthetic material may not be available in some centers and its use is associated with a high risk of infection, thrombosis, aneurysmal dilatation or late rejection [5]. The autologous peritoneal patch or peritoneal tube is technically easy to prepare and use, with no need for another incision and is an alternative to prosthetic material or autologous veins [6].

The use of autologous peritoneal patch is a good solution for repairing vascular defects that are not amenable to primary repair [7]. These are easily accessible, adjustable to the required size, free of charge and low risk of infection and thrombosis.

\section{Objective}

Presentation a clinical case of an advanced locally adenocarcinoma of the head of the pancreas with a partial resection of the inferior vena cava, reconstructed with autologous peritoneum patch.

\section{Material and methods}

Patient with locally advanced head adenocarcinoma of the pancreas at imaging staging, initially submitted to bilio-digestive shunt at another institution. The patient was referred to our Hepatobiliary Pancreatic Center (CHBP), discussed at a multidisciplinary meeting and whose decision was neoadjuvant chemotherapy. After completing 4 cycles of FOLFIRINOX, it was re-staged clinically, analytically and imagiologically with thoraco-abdomino-pelvic CT-angiography, being re-discussed in a multidisciplinary meeting. Categorized according to RECIST criteria as RP, with a positive response and size decrease of at least $30 \%$. It was proposed for exploratory Laparotomy and according to intraoperative evaluation, possible a Cephalic Duodenopancreatectomy. In the intraoperative it was verified the absence of disease at distance. Surgical resection implied a $4 \mathrm{~cm}$ resection of the anterior face of the inferior vena cava to the level of emergence of the left renal vein due to adhesion / tumor invasion. The reconstruction was performed with parietal peritoneal patch. The patch was resected and placed in saline solution. After isolation and venous vascular exclusion, was done a primary suture of the patch with prolene ${ }^{\circledR}$ 5.0. After disclamping, excellent flow, with maintained vascular patency. The surgery was uneventful, with no need for blood products consumption and with hemodynamic stability (Figure 1).
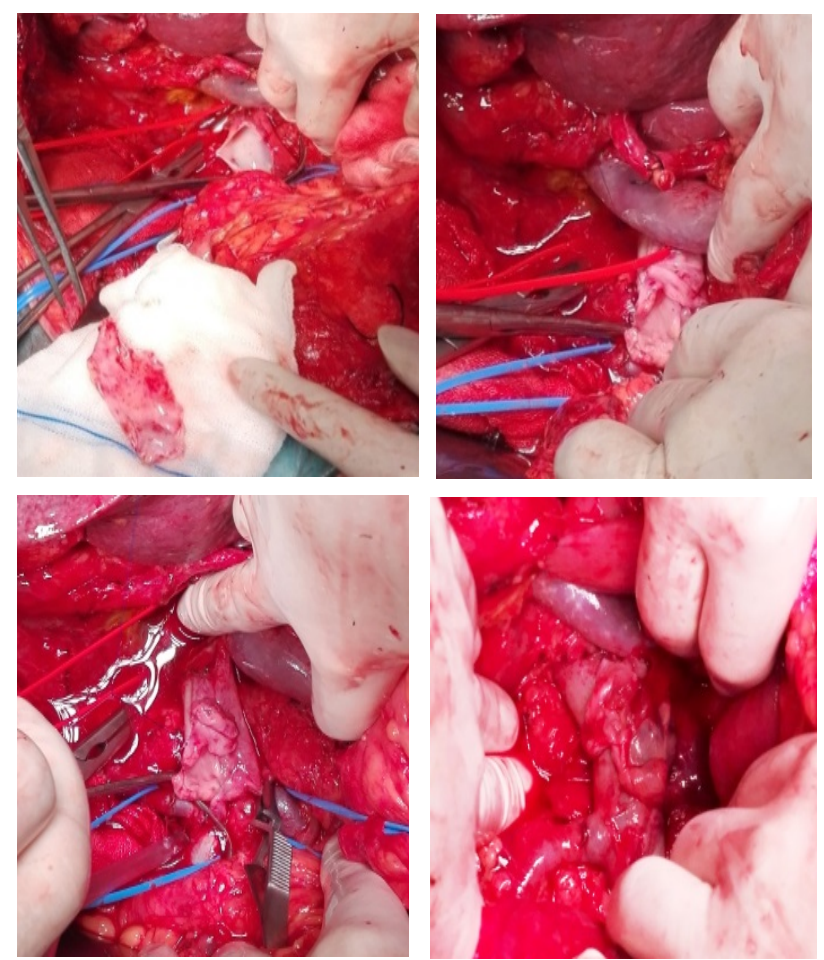

Figure 1. Reconstruction with a parietal peritoneal patch

${ }^{*}$ Correspondence to: Catarina Aguiar, CHBPT, Centro Hepato-Bilio-Pancreatico e Transplantação, Centro Hospitalar Lisboa Central, Lisbon, Portugal, E-mail: Catarina_Aguiar_99@hotmail.com

Key words: peritoneal patch, vascular reconstruction, oncological vascular ressection, pancreaticoduodenectomy

Received: January 03, 2019; Accepted: January 12, 2019; Published: January 14, 2019 


\section{Results}

Adequate reconstructed vena cava blood flow confirmed by Eco-Doppler on the 2nd postoperative day and by Angio-CT on the 4 th postoperative day. Post-operative without morbidity, as infection, pancreatic fistula or venous thrombosis. In the postoperative period, the patient was not medicated with anticoagulants or antiaggregators. Histologically, it has been confirmed that it is a moderately differentiated pancreatic adenocarcinoma of the pancreas, pT3N1M0R1. The patient was followed up at our Hepatobiliary Pancreatic Center (CHBP), and at 6 months postoperatively, he was clinically well, without signs of imaging relapse and without expressing tumor markers. He underwent adjuvant chemotherapy until the 8th postoperative month and on the CT scan control, did not show any evidence of disease progression, with normal tumor markers. At 22 months of follow-up, the patient is alive, clinically well and without evidence of disease.

\section{Conclusion}

Knowing that the only cure for pancreatic adenocarcinoma is Surgery, autologous peritoneal patch (peritoneal tube patch or a simple patch) represents a safe, low-cost, easily accessible option, avoiding the disadvantages and complications of synthetic prosthesis, allowing the resection of locally advanced tumors.

\section{References}

1. Ferlay J, Shin HR, Bray F, Forman D, Mathers C, et al. (2010) GLOBOCAN 2008v2.0, Cancer Incidence and Mortality Worlswide. International Agency for Research on Cancer.

2. Cartwright T, Richards DA, Boehm KA (2008) Cancer of the pancreas are we making a progress? A review of studies in the US Oncology Research Network. Cancer Control 15: 307-313. [Crossref]

3. Yoshioka M, Onda M, Tajiri T, Akimaru K, et al. (2001) Reconstruction of the portal vein using a peritoneal patch-graft Am J Surg 181: 247-250. [Crossref]

4. Yaita A, Nakamura T, Sugimachi K, Inokuchi K et al. (1975) Use of free peritoneal patch in reenforcing alimentary tract anastomosis Jpn J Surg 5: 56-63. [Crossref]

5. MasatoYoshioka, Masahiko Onda, Takashi Tajiri, Koho Akimaru, ShoMineta et al (2001) Reconstruction of the portal vein using a peritoneal patch-graft The American Journal of Surgery Volume 181: 247-250. [Crossref]

6. Sabuncuoglu MZ, Dandin O, Teomete U, Cakir T, Kayaalp (2015) Hippokratia Using autologous peritoneal graft for portal vein injury due to blunt thoracoabdominal trauma. 19: 260-262. [Crossref]

7. Kurumboor Prakash, Jean-Marc Regimbeau, Jacques Belghiti (2003) Reconstruction of portal vein using a hepatic vein patch graft after combined hepatectomy and portal vein resection The American Journal of Surgery 185: 230-231. [Crossref]

Copyright: (C2019 Aguiar C. This is an open-access article distributed under the terms of the Creative Commons Attribution License, which permits unrestricted use, distribution, and reproduction in any medium, provided the original author and source are credited. 